\title{
Variación estacional de la avifauna del Bosque Montano en la Quebrada del Portugués, Tucumán, Argentina
}

\author{
Bird Seasonal Variation of the Montane Forest in the Quebrada \\ del Portugués, Tucumán, Argentina
}

\author{
María E. Fanjul1,2,; Claudia M. Antelo1; Zulma J. Brandán Fernández'; \\ María V. Martínez;; Ada L. Echevarria'; Nora L. Marigliano'; Celina I. Navarro ${ }^{\dagger}$ \\ 1 Fundación Miguel Lillo, Miguel Lillo 251, (4000) San Miguel de Tucumán, Tucumán, Argentina. \\ 2 Facultad de Ciencias Naturales e IML, Universidad Nacional de Tucumán, Miguel Lillo 205, (4000) \\ San Miguel de Tucumán, Tucumán, Argentina. \\ *mefanjul@lillo.org.ar.
}

\section{RESUMEN}

La Quebrada del Portugués es un Área de Importancia para la Conservación de las Aves (AICA) y forma parte del Parque Nacional Aconquija. El sector estudiado corresponde al Distrito de los Bosques Montanos de las Yungas. El objetivo fue conocer la composición y variación estacional del ensamble de aves que frecuentan un sector de dicha área. Se realizaron 161 muestreos de las aves vistas u oídas, en puntos de radio fijo de $30 \mathrm{~m}$ y 15 minutos de duración, entre agosto y noviembre de 2014 y entre febrero y junio de 2015. Se encontraron 93 especies de 31 familias y 16 órdenes. La riqueza por estación fue de 72 especies para el período seco y de 60 especies para el período húmedo. Las especies más abundantes a lo largo del año fueron Zonotrichia capensis, Turdus chiguanco, Mecocerculus leucoprhrys, Cypseloides rothschildi y Vanellus chilensis. Seis especies, consideradas Amenazadas a Nivel Mundial, fueron registradas: Vultur gryphus, Cypseloides rothschildi, Scytalopus superciliaris, Elaenia strepera, Cinclus schulzii y Atlapetes citrinellus. A partir de este primer relevamiento de un sector de Bosques Montanos, se daría indicio del potencial biológico que tiene esta zona para toda la región, sumado al hecho de que la misma forma parte del nuevo Parque Nacional Aconquija. Sería importante realizar estudios estacionales a largo

\footnotetext{
> Ref. bibliográfica: Fanjul, M. E.; Antelo, C. M.; Brandán Fernández, Z. J.; Martínez, M. V.; Echevarria, A. L.; Marigliano, N. L.; Navarro, C. I. 2019. “Variación estacional de la avifauna del bosque montano en la Quebrada del Portugués, Tucumán, Argentina". Acta zoológica lilloana 63 (2): 33-47. Fundación Miguel Lillo, Tucumán, Argentina. D.O.I.: https://doi.org/10.30550/j.azl/2019.63.2/3

Recibido: 11/06/19 - Aceptado: 15/10/19

> URL de la revista: http://actazoologica.lillo.org.ar

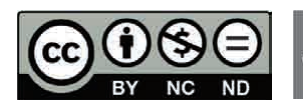

> Algunos derechos reservados. Esta obra está bajo una Licencia Creative Commons Atribución - No Comercial - Sin Obra Derivada 4.0 Interna- 
plazo en sus diferentes ambientes para promover un adecuado plan de manejo y conservación.

Palabras clave - AICA, Yungas, aves, riqueza, estacionalidad.

\section{ABSTRACT}

The Quebrada del Portugués is an Important Bird Area (IBA) and is part of the Aconquija National Park. The sector studied corresponds to the District of the Montane Forests in the Yungas Ecoregion. The aim of this work was to describe the composition and seasonal structure of the bird assemblage that frequent a sector of that area. The 161 samplings were made at fixed radio points of $30 \mathrm{~m}$ during 15 minutes, where all the species seen or heard were recorded, from August to November 2014 and from February to June 2015. A total of 93 species from 31 families and 16 orders were registered. The seasonal richness was 72 species for the dry period and 60 for the wet period. The most abundant species along the year were Zonotrichia capensis, Turdus chiguanco, Mecocerculus leucoprhrys, Cypseloides rothschildi and Vanellus chilensis. Six species observed are considered Globally Endangered: Vultur gryphus, Cypseloides rothschildi, Scytalopus superciliaris, Elaenia strepera, Cinclus schulzii and Atlapetes citrinellus. This survey in a Montane Forest area highlights the biological potential that this protected area has for the entire region considering the recent creation of the new Aconquija National Park. It would be important to carry out seasonal, long-term studies in their different environments, to promote an adequate management and conservation planning.

Keywords - IBA, Yunas, birds, richness, seasonality.

\section{INTRODUCCIÓN}

Los Bosques Montanos de Sudamérica son objeto de interés mundial debido a la particular biodiversidad que presentan, las especies endémicas, sus bienes y servicios ecosistémicos y su fragilidad ante el avance de la frontera agropecuaria (Chalukian, 1991; Malizia, Pacheco, Blundo, Brown, 2012; Tejedor Garavito et al., 2012). Pertenecen a la Provincia Fitogeográfica de las Yungas la cual se caracteriza por un marcado gradiente de riqueza de especies tanto a nivel latitudinal como altitudinal, siendo los Bosques Montanos el piso de menor riqueza (Brown, Pacheco, Lomáscolo, Malizia, 2006). Dichos bosques, en el noroeste de Argentina, se desarrollan en los faldeos orientales de las Sierras Subandinas y Sierras Pampeanas, sobre pequeños valles y zonas de quebradas (Brown, Grau, Malizia, Grau, 2001; Barros y Giménez, 2017). Son formaciones con un fuerte impacto antrópico, con ganadería de trashumancia y quema de pastizales, así como también desplazamientos de laderas (Ahumada, Ledesma, Ibañez Palacio, Delgado, Jiménez, 2000; Grau, 2005). 
La Quebrada del Portugués, ubicada en la provincia de Tucumán, forma parte de un área protegida que recientemente se estableció como Parque Nacional Aconquija e integra un importante núcleo de conservación (Chiesa, 2018). Ornitológicamente es considerada un Área de Importancia para la Conservación de las Aves (AICA) y en ella se pueden encontrar ensambles de aves típicas de las Yungas, de matorrales montanos y de ambientes andinos puneños (Moschione, 2005). Algunas de las especies citadas para la zona tienen prioridad de conservación por su rango restringido de distribución como Amazona tucumana, Cypseloides rothschildi, Scytalopus supercilliaris, Atlapetes citrinellus, Cinclus schulzi y Eriocnemis glaucopoides (Stattersfield, Crosby, Long, Wege, 1998).

Por lo general, la composición de la avifauna en los distintos ambientes, depende de diferentes factores que actúan tanto a escala temporal como espacial (Wiens, 1992; Echevarria y Fanjul, 2016; Santillán et al., 2018). A escala temporal, estos cambios pueden estar asociados a fluctuaciones en la disponibilidad de alimento (Echevarria y Fanjul, 2016), al arribo, partida y/o establecimiento de especies migratorias (Rappole, 1995). La estacionalidad de los ensambles de aves está altamente relacionada con la precipitación y cambios hidrológicos a escala local, mientras que variaciones interanuales pueden depender de eventos macroclimáticos que operan a escala global y regional (Fava, Acosta, Blanco, 2017).

Sobre la avifauna de los Bosques Montanos en la provincia de Tucumán, solamente se cuenta con los relevamientos de Vides Almonacid $(1985,1992)$ los cuales mencionan un total de 73 especies y una marcada estacionalidad en el ensamble, caracterizada por el arribo de especies migrantes y poblaciones con desplazamientos altitudinales. Existen antecedentes sobre la avifauna presente en áreas protegidas próximas a la zona de estudio y que actualmente integran el nuevo Parque Nacional Aconquija (Malizia, Aragón, Chacoff, Monmany, 1998; Pérez Miranda, 2003; Cocimano, Chani, Echevarria, Marano, 2005; Navarro, Brandán, Marigliano, 2007; Echevarria, 2014) pero no son estudios específicos para el distrito de Bosques Montanos de la zona. Por lo tanto, nuestros objetivos fueron describir y analizar la composición y variación estacional de la comunidad de aves de un sector del Bosque Montano en la Quebrada del Portugués. Por último, con los resultados obtenidos se pretende generar información de base sobre la avifauna de la zona, útil para diseñar futuras investigaciones y planificar medidas efectivas de manejo.

\section{MATERIALES Y MÉTODOS}

\section{Área de estudio}

Desde la localidad de El Rincón, Departamento Tafí del Valle (Tucumán), se recorrieron caminando $10 \mathrm{Km}$ hacia el interior de la Quebrada del Portugués, por la traza de la Ruta provincial No 325 . La quebrada está limitada al oeste por la Sierra del Aconquija y al este por los Cerros Nuñorco. La zona de estudio se encuentra entre $\operatorname{los} 26^{\circ} 58^{\prime} \mathrm{S}, 65^{\circ} 44^{\prime} \mathrm{W}$ y $27^{\circ} 08^{\prime} \mathrm{S}, 65^{\circ} 45^{\prime} \mathrm{W}$ (Figura 1). Los Bosques Montanos se distribuyen en el piso altitudinal que va desde los 1600 a 2500 m s.n.m. aproximadamente (Cabrera, 1976; Pérez Miranda, 2003) y presentan diferentes unidades de 


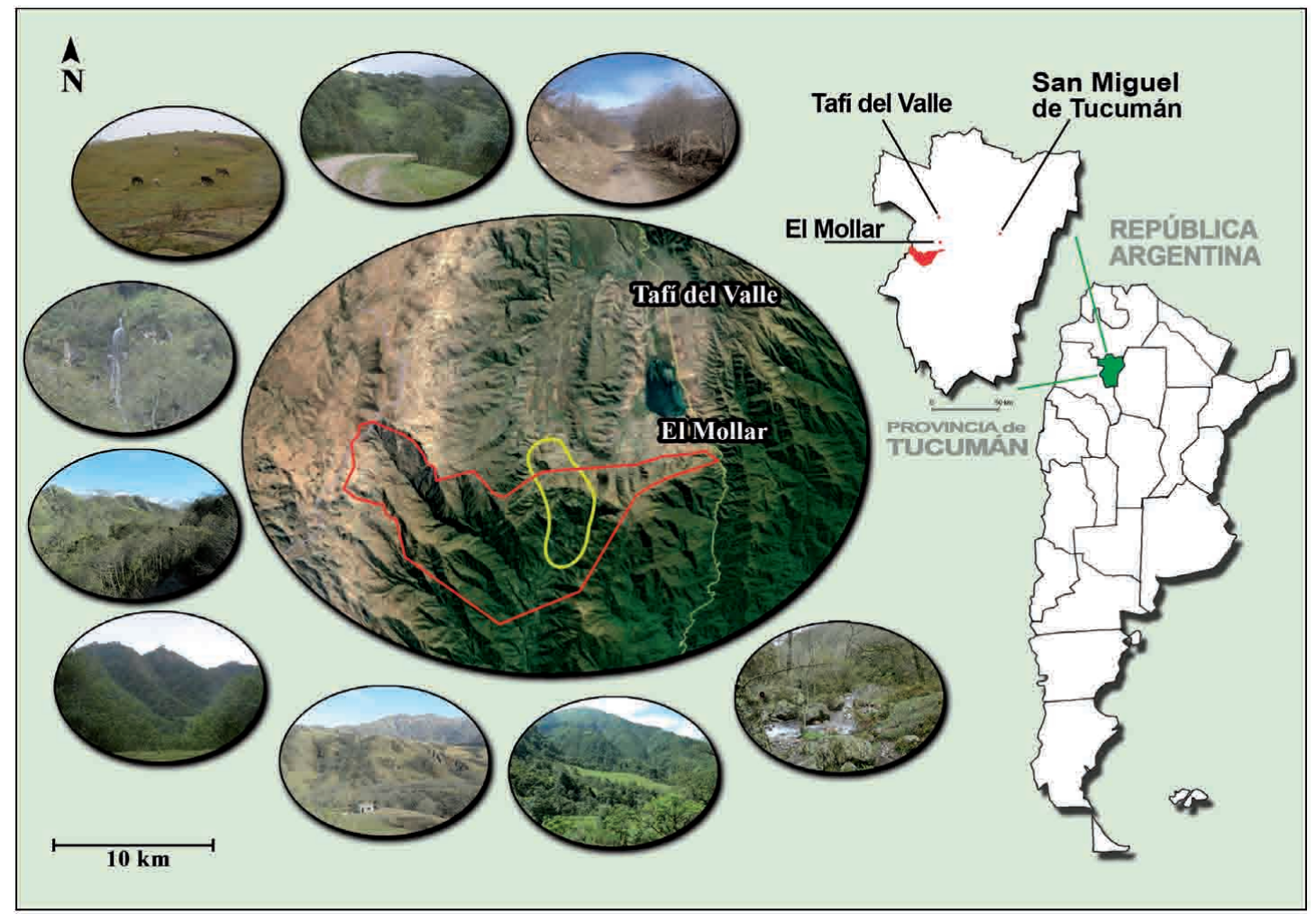

Figura 1. Ubicación, en amarillo, del área de estudio. En rojo, Reserva Provincial Quebrada del Portugués, provincia de Tucumán, Argentina.

vegetación, como pastizales, arbustales y bosques simples de Alnus acuminata, Podocarpus parlatorei, Sambucus peruviana y Polylepis australis (Arturi, Grau, Aceñolaza, Brown, 1998). El clima de la zona es de tipo continental y subtropical de serranía, marcadamente estacional, con un período cálido y húmedo, con temperaturas medias de $13-14^{\circ} \mathrm{C}$ en primavera-verano y otro templado-frío y seco, con temperaturas medias de $5-6^{\circ} \mathrm{C}$ en otoño-invierno (Bianchi y Cravero, 2010). Los puntos de observación de aves se establecieron en zonas de bosques de aliso (Alnus acuminata), especie dominante de este sector, que en algunas laderas estuvo acompañado por Ilex argentina, Duranta serratifolia, Sambucus peruviana y en los estratos bajos por Salvia elegans, Urtica sp. y Pteris deflexa.

\section{Muestreo de la avifauna}

La nomenclatura y clasificación taxonómica se realizó en base a los criterios de Remsen et al., (2019). Se efectuaron campañas de muestreo desde agosto a noviembre de 2014 y desde febrero a junio de 2015. Los muestreos de aves se efectuaron siguiendo el método de puntos de radio fijo de $30 \mathrm{~m}$ y 15 minutos de duración, con una distancia media entre ellos de al menos 500 m (Bibby, Hill, Burgess Bibby, 2000). Se registraron todas las especies e individuos vistos u oídos, perchados, volando o realizando cualquier actividad, durante las primeras horas de la mañana, con un total de 161 muestras independientes en tiempo y espacio, abarcando dos días una vez al 
mes en cada período, seco (otoño-invierno, $\mathrm{N}=88$ ) y húmedo (primavera-verano, $\mathrm{N}=73$ ). Siguiendo a Bibby et al., (2000), no se realizaron muestreos en condiciones climáticas adversas de vientos fuertes, niebla o lluvias.

\section{Análisis de datos}

Se consideró el número de especies presentes para toda la comunidad (Riqueza Total) y para cada período (Riqueza Estacional). La abundancia relativa (AR) para cada especie se calculó como la relación porcentual del número de individuos de la especie registrada en todos los censos $\left(\mathrm{n}_{\mathrm{i}}\right)$ en relación al total de individuos de todas las especies por estación $(\mathrm{N})$, destacándose sólo a las especies con AR mayor o igual a 9.

Para comparar las medianas de riqueza y abundancia relativa entre los diferentes períodos del año se realizó un análisis no paramétrico (Test Mann Whitney). Los análisis fueron realizados con el programa InfoStat 2018, con un nivel de significancia de $\alpha=0,05$.

Para evaluar la composición de la comunidad entre los dos períodos, se calculó: el índice de similitud de Jaccard (Magurran y McGill, 2011), con los datos la presencia-ausencia de las especies en los diferentes períodos evaluados y la Similitud Proporcional (SP) entre los períodos (Feinsinger, 2003), con los datos de abundancia relativa SP se obtiene en función de los valores de $p_{i}\left(p_{i}=n_{i} / N\right.$; donde $n_{i}$ es el número de individuos de la especie i y $\mathrm{N}$ es el número total de individuos), para las especies en cada una de las dos muestras. Además, para evaluar la diversidad de la comunidad de aves entre los períodos seco y húmedo se graficó las curvas de rangoabundancia, que destacan los cambios en el orden de abundancia de las especies y la variación entre los períodos en cuanto a la dominancia numérica (Feinsinger, 2003) y se obtuvo el Índice de Simpson Inverso $\left(\mathrm{C}_{\text {inv }}\right)$ (Feinsinger, 2003) de las aves en cada período. $\mathrm{C}_{\text {inv }}$ tiene un valor mínimo de 1 (para muestras con una sola especie) y un valor máximo que equivale al número total de especies cuando todas las especies tienen exactamente la misma abundancia en un período dado. $\mathrm{C}_{\mathrm{inv}}$ es más sensible a los cambios en la igualdad de especies que, por ejemplo, el Índice de Shannon-Weaver (Feinsinger, 2003). Luego se realizó un Test de t para determinar si los valores de los índices eran estadísticamente diferentes o no (Magurran y McGill, 2011).

Las especies migratorias se definieron según su lugar de nidificación en: migrantes regionales (MR), especies que nidifican en la Patagonia, centro, noreste de Argentina y visitan el área de estudio en diferentes estaciones y migrantes altitudinales (MA), especies que nidifican en la alta montaña y en otoño se desplazan a zonas de menor altitud (Olrog, 1979; Canevari et al., 1991; Capllonch, 1997; Mazar Barnett y Pearman, 2001; Capllonch y Lobo, 2005; Capllonch, Ortiz, Soria, 2008; Echevarria, Marano, Chani, Cocimano, 2008; Antelo y Brandán, 2013, Ortiz et al., 2013).

Para el estatus de conservación a nivel internacional se consultó los apéndices de la Convención sobre el Comercio Internacional de Especies Amenazadas de Fauna y Flora Silvestre (CITES, 2017), la Lista Roja de la Unión Internacional para la Conservación de la Naturaleza (UICN, 2018) y a nivel nacional la categorización de López Lanús, Grilli, Coconier, Di Giacomo, Banchs (2008). 


\section{RESULTADOS}

Registramos en total 1701 individuos pertenecientes a 93 especies, 31 familias y 16 órdenes (Tabla 1). La riqueza estacional de especies fue de 72 para el período seco y 60 para el período húmedo. Según el análisis no paramétrico U de Mann-Whitney, el número de especies no presentó diferencias significativas entre los períodos, seco (Media $\pm \mathrm{SD}=3,85 \pm 1,78)$ y húmedo (Media $\pm \mathrm{SD}=3,82 \pm 1,86$ ): $\mathrm{W}=5816$; $p=0,74$.

La abundancia de especies fue de 932 individuos para el período seco y 769 para el período húmedo, donde se observaron diferencias estadísticamente significativas para la abundancia relativa entre el período seco (Media $\pm \mathrm{SD}=0,07 \pm 0,09$ ) y el húmedo (Media $\pm \mathrm{SD}=0,04 \pm 0,04)$ : $\mathrm{W}=5328 ; p=0,05$. Las especies que presentaron los mayores valores de AR (mayor o igual a 9) en el período seco fueron Zonotrichia capensis $(\mathrm{AR}=21,46)$, Turdus chiguanco $(\mathrm{AR}=15,66)$ y Vanellus chilensis $(\mathrm{AR}=9,87)$; en tanto que en el período húmedo el mayor registro fue de Cypseloides rothschildi $(\mathrm{AR}=15,71)$, seguidos por Turdus chiguanco $(\mathrm{AR}=11)$, Streptoprocne zonaris $(\mathrm{AR}=9,82)$ y Zonotrichia capensis $(\mathrm{AR}=9,03)$ (Tabla 1$)$.

Según el índice de Jaccard, la similitud en riqueza de especies entre los períodos evaluados fue de 59\%. El índice SP dio que la composición y proporción de individuos de las especies entre ambos períodos fue de un $51 \%$.

El Índice de diversidad de Simpson Inverso dio para el período seco $\mathrm{C}_{\mathrm{inv}}=$ 10,98 y para el período húmedo $C_{\text {inv }}=14,23$, los cuales no fueron significativamente diferentes $(T=7,76 ; p=0,082)$. Las curvas de rango-abundancia mostraron (Figura 2) que, por el ancho del gráfico sobre el eje $X$, el cual refleja el número de especies presente en cada período, se puede observar que la curva del período seco abarca un ancho mayor y por lo tanto tiene una riqueza de especies mayor que el período húmedo. También se nota que esta diferencia es dada por la longitud de las colas, que indican una alta presencia de especies con baja abundancia. Además, en función del largo y forma de las curvas, que indican el grado de igualdad o dominancia numérica, ambos períodos son similares. Sin embargo, la secuencia de las especies es diferente, ya que la dominancia numérica de estas varía entre los períodos. Para el seco se destacan Zonotrichia capensis, Turdus chiguanco y Vanellus chilensis y para el húmedo las especies dominantes son Cypseloides rothschildi, Turdus chiguanco, Streptoprocne zonaris, Zonotrichia capensis, Mecocerculus leucophrys y Pygochelidon cyanoleuca. Esto coincide con los valores observados de AR para cada período.

Se encontraron 12 especies migratorias, cuatro altitudinales y ocho regionales (Tabla 1). Del total de especies registradas, las migratorias representaron el $12,90 \%$ de las especies de la comunidad.

Según el estatus de conservación se identificó una especie presente en el Apéndice I y 17 especies en el Apéndice II, de CITES, 2017 (Tabla 1). Se registraron especies que a nivel global, la UICN (2018) las considera como «Vulnerables»: Cinclus schulzii y Vultur gryphus y «En riesgo»: Cypseloides rothschildi. Además, a nivel nacional, López Lanús et al. (2008) las clasifica en la categoría de «Amenazada»: Buteo albigula, Atlapetes citrinellus y Cinclus schulzii, como «Vulnerables»: Vultur gryphus y Spizaetus melanoleucus y como «Insuficientemente conocido su estado» Cypseloides rothschildi. 
Tabla 1. Lista de especies registradas en la Quebrada del Portugués, Argentina. Especies migratorias: * migrantes regionales, ** migrantes altitudinales. Abundancia Relativa en Estación Seca (AR Seca) y Abundancia Relativa en Estación Húmeda (AR Húmeda). Categorías de (CITES I y II); IUCN: Vu (vulnerable), ER (en riesgo) y PM (preocupación menor). Nomenclatura de Remsen et al., 2019. Código referencia para Figura 2. SD (Sin Datos).

\begin{tabular}{|c|c|c|c|c|c|}
\hline Familia & Especie/Especie migratoria/Código & $\begin{array}{c}\text { AR } \\
\text { Seca }\end{array}$ & $\begin{array}{c}\text { AR } \\
\text { Húmeda }\end{array}$ & CITES & IUCN \\
\hline Anatidae & Anas flavirostris / */ ANAFL & 0,215 & SD & SD & PM \\
\hline \multirow[t]{5}{*}{ Columbidae } & Columba livia / COLLI & 0,107 & SD & SD & PM \\
\hline & Zenaida auriculata / ZENAU & 0,429 & SD & SD & PM \\
\hline & Columbina picui / COLPI & 1,180 & SD & SD & PM \\
\hline & Leptotila megalura / LEPME & 0,322 & 0,916 & SD & PM \\
\hline & Leptotila verreauxi / LEPVE & 0,429 & SD & SD & PM \\
\hline Cuculidae & Tapera naevia / */ TAPNA & SD & 0,131 & SD & PM \\
\hline \multirow[t]{2}{*}{ Apodidae } & Cypseloides rothschildi / CYPRO & SD & 15,707 & SD & ER \\
\hline & Streptoprocne zonaris / **/ STRZO & SD & 9,817 & SD & PM \\
\hline \multirow[t]{5}{*}{ Trochilidae } & Adelomyia melanogenys / ADEME & SD & 0,131 & CITES II & PM \\
\hline & Sappho sparganurus / **/ SAPSP & 0,536 & 1,963 & CITES II & PM \\
\hline & Chlorostilbon lucidus / CHLLU & 0,000 & 0,131 & CITES II & PM \\
\hline & Amazilia chionogaster / AMACH & SD & 0,393 & CITES II & PM \\
\hline & Hylocharis chrysura / HYLCH & 0,107 & SD & CITES II & PM \\
\hline Rallidae & Pardirallus sanguinolentus / PARSA & 0,107 & SD & SD & PM \\
\hline Charadriidae & Vanellus chilensis / VANCH & 9,871 & 2,880 & SD & PM \\
\hline Ardeidae & Egretta thula /EGRTH & SD & 0,262 & SD & PM \\
\hline \multirow[t]{2}{*}{ Cathartidae } & Vultur gryphus / VULGR & 2,575 & 0,916 & CITES I & $\mathrm{Vu}$ \\
\hline & Cathartes aura / CATAU & SD & 0,262 & CITES II & PM \\
\hline \multirow[t]{6}{*}{ Accipitridae } & Spizaetus melanoleucus / SPIME & 0,215 & SD & CITES II & PM \\
\hline & Buteogallus urubitinga / BUTUR & 0,107 & 0,131 & CITES II & PM \\
\hline & Rupornis magnirostris / RUPMA & 0,107 & SD & CITES II & PM \\
\hline & Geranoaetus melanoleucus / GERME & 0,322 & 0,131 & CITES II & PM \\
\hline & Geranoaetus polyosoma / GERPO & 0,322 & SD & CITES II & PM \\
\hline & Buteo albigula / BUTAL & 0,107 & SD & CITES II & PM \\
\hline Strigidae & Athene cunicularia / ATHCU & 3,648 & 0,393 & CITES II & PM \\
\hline Alcedinidae & Megaceryle torquata / MEGTO & 0,107 & SD & SD & PM \\
\hline \multirow[t]{4}{*}{ Picidae } & Colaptes rubiginosus / COLRU & 0,215 & SD & SD & PM \\
\hline & Colaptes melanochloros / COLME & 0,429 & SD & SD & PM \\
\hline & Colaptes rupicola / COLRU & 2,468 & 0,262 & SD & PM \\
\hline & Campephilus leucopogon / CAMLE & 0,644 & 0,131 & SD & PM \\
\hline Cariamidae & Cariama cristata / CARCR & 0,429 & 0,916 & SD & PM \\
\hline \multirow[t]{3}{*}{ Falconidae } & Caracara plancus / CARPL & 4,399 & 4,188 & CITES II & PM \\
\hline & Milvago chimango / MILCH & 1,073 & 0,916 & CITES II & PM \\
\hline & Falco sparverius / FALSP & 1,288 & 0,654 & CITES II & PM \\
\hline Psittacidae & Pionus maximiliani / PIOMA & 0,215 & 0,654 & CITES II & PM \\
\hline Rhinocryptidae & Scytalopus superciliaris / SCYSU & 0,107 & SD & SD & PM \\
\hline \multirow[t]{8}{*}{ Furnariidae } & Geositta tenuirostris / GEOTE & 0,215 & SD & SD & PM \\
\hline & Sittasomus griseicapillus / SITGR & 0,322 & SD & SD & PM \\
\hline & Cinclodes fuscus / CINFU & 0,322 & SD & SD & PM \\
\hline & Cinclodes atacamensis / CINAT & 1,717 & 0,785 & SD & PM \\
\hline & Syndactyla rufosuperciliata / SYNRU & 0,215 & 0,131 & SD & PM \\
\hline & Leptasthenura fuliginiceps / LEPFU & 0,858 & 0,131 & SD & PM \\
\hline & Leptasthenura aegithaloides / LEPAE & 0,215 & SD & SD & PM \\
\hline & Synallaxis frontalis / SYNFR & 0,429 & SD & SD & PM \\
\hline
\end{tabular}


Tabla 1 (cont.).

\begin{tabular}{|c|c|c|c|c|c|}
\hline Familia & Especie/Especie migratoria/Código & $\begin{array}{c}\text { AR } \\
\text { Seca }\end{array}$ & $\begin{array}{c}\mathrm{AR} \\
\text { Húmeda }\end{array}$ & CITES & IUCN \\
\hline \multirow[t]{11}{*}{ Tyrannidae } & Elaenia albiceps / */ ELAAL & 0,215 & 0,393 & SD & PM \\
\hline & Elaenia parvirostris / ELAPA & 0,322 & 0,524 & SD & PM \\
\hline & Elaenia strepera / ELAST & 0,322 & 0,131 & SD & PM \\
\hline & Mecocerculus leucophrys / MECLE & 4,828 & 8,246 & SD & PM \\
\hline & Phaeomyias murina / PHAMU & SD & 0,262 & SD & PM \\
\hline & Knipolegus cabanisi / KNICA & SD & 0,262 & SD & PM \\
\hline & Knipolegus aterrimus / * KNIAT & 0,429 & 0,916 & SD & PM \\
\hline & Myiotheretes striaticollis / MYIST & 0,644 & 0,916 & SD & PM \\
\hline & Myiodynastes maculatus / */ MYIMA & 0,107 & SD & SD & PM \\
\hline & $\begin{array}{l}\text { Empidonomus aurantioatrocristatus / } \\
\text { */ EMPAU }\end{array}$ & SD & 0,393 & SD & PM \\
\hline & Myiarchus tyrannulus / MYITY & 0,107 & 0,524 & SD & PM \\
\hline Tityridae & Pachyramphus validus / PACVA & SD & 1,047 & SD & PM \\
\hline \multirow[t]{2}{*}{ Hirundinidae } & Pygochelidon cyanoleuca / PYGCY & SD & 6,675 & SD & PM \\
\hline & Tachycineta leucopyga / */ TACLEp & SD & 0,524 & SD & PM \\
\hline \multirow[t]{2}{*}{ Troglodytidae } & Troglodytes aedon / TROAE & 1,073 & 0,524 & SD & PM \\
\hline & Troglodytes solstitialis / TROSO & 1,073 & 0,785 & SD & PM \\
\hline Cinclidae & Cinclus schulzii / CINSC & 0,215 & SD & SD & $\mathrm{Vu}$ \\
\hline \multirow[t]{4}{*}{ Turdidae } & Turdus rufiventris / TURRU & 0,429 & 0,262 & SD & PM \\
\hline & Turdus amaurochalinus / TURAM & 0,215 & 0,654 & SD & PM \\
\hline & Turdus nigriceps / TURNI & SD & 0,131 & SD & PM \\
\hline & Turdus chiguanco /TURCH & 15,665 & 10,995 & SD & PM \\
\hline \multirow[t]{11}{*}{ Thraupidae } & Thlypopsis ruficeps / THLRU & SD & 0,393 & SD & PM \\
\hline & Pipraeidea melanonota / PIPME & 0,107 & 0,131 & SD & PM \\
\hline & Pipraeidea bonariensis / PIPBO & 0,000 & 0,262 & SD & PM \\
\hline & Thraupis sayaca / THRSA & 0,536 & SD & SD & PM \\
\hline & Phrygilus carbonarius / PHRCA & SD & 0,131 & SD & PM \\
\hline & Poospiza hypochondria / $\mathrm{POOHY}$ & SD & 0,131 & SD & PM \\
\hline & Microspingus erythrophrys / MICER & 1,180 & SD & SD & PM \\
\hline & Sicalis flaveola / SICFL & 0,215 & 0,131 & SD & PM \\
\hline & Sicalis luteola / SICLU & 0,107 & 0,000 & SD & PM \\
\hline & Catamenia analis / */ CATAN & SD & 0,262 & SD & PM \\
\hline & Saltator aurantiirostris / SALAU & 0,215 & SD & SD & PM \\
\hline \multirow[t]{5}{*}{ Emberizidae } & Chlorospingus flavopectus / CHLFL & 0,966 & SD & SD & PM \\
\hline & Rinchospiza strigiceps / RINST & 1,073 & SD & SD & PM \\
\hline & Arremon flavirostris / ARRFL & 0,322 & SD & SD & PM \\
\hline & Zonotrichia capensis / ZONCA & 21,459 & 9,031 & SD & PM \\
\hline & Atlapetes citrinellus / ATLCI & 4,506 & 2,094 & SD & PM \\
\hline Cardinalidae & Pheucticus aureoventris / PHEAU & 0,751 & 1,440 & SD & PM \\
\hline \multirow[t]{3}{*}{ Parulidae } & Setophaga pitiayumi / **/ SETPI & SD & 0,524 & SD & PM \\
\hline & Basileuterus culicivorus / BASCU & 0,751 & 0,785 & SD & PM \\
\hline & Myioborus brunniceps / **/ MYIBR & 0,966 & 1,571 & SD & PM \\
\hline \multirow[t]{4}{*}{ Icteridae } & Cacicus chrysopterus / CACCH & 0,322 & SD & SD & PM \\
\hline & Icterus pyrrhopterus / ICTPY & 0,107 & SD & SD & PM \\
\hline & Agelaioides badius / AGEBA & 0,215 & SD & SD & PM \\
\hline & Molothrus bonariensis / MOLBO & 0,536 & 1,702 & SD & PM \\
\hline Fringilidae & Spinus magellanicus / SPIMA & 3,112 & 3,927 & SD & PM \\
\hline Passeridae & Passer domesticus / PASDO & 0,429 & SD & SD & PM \\
\hline
\end{tabular}




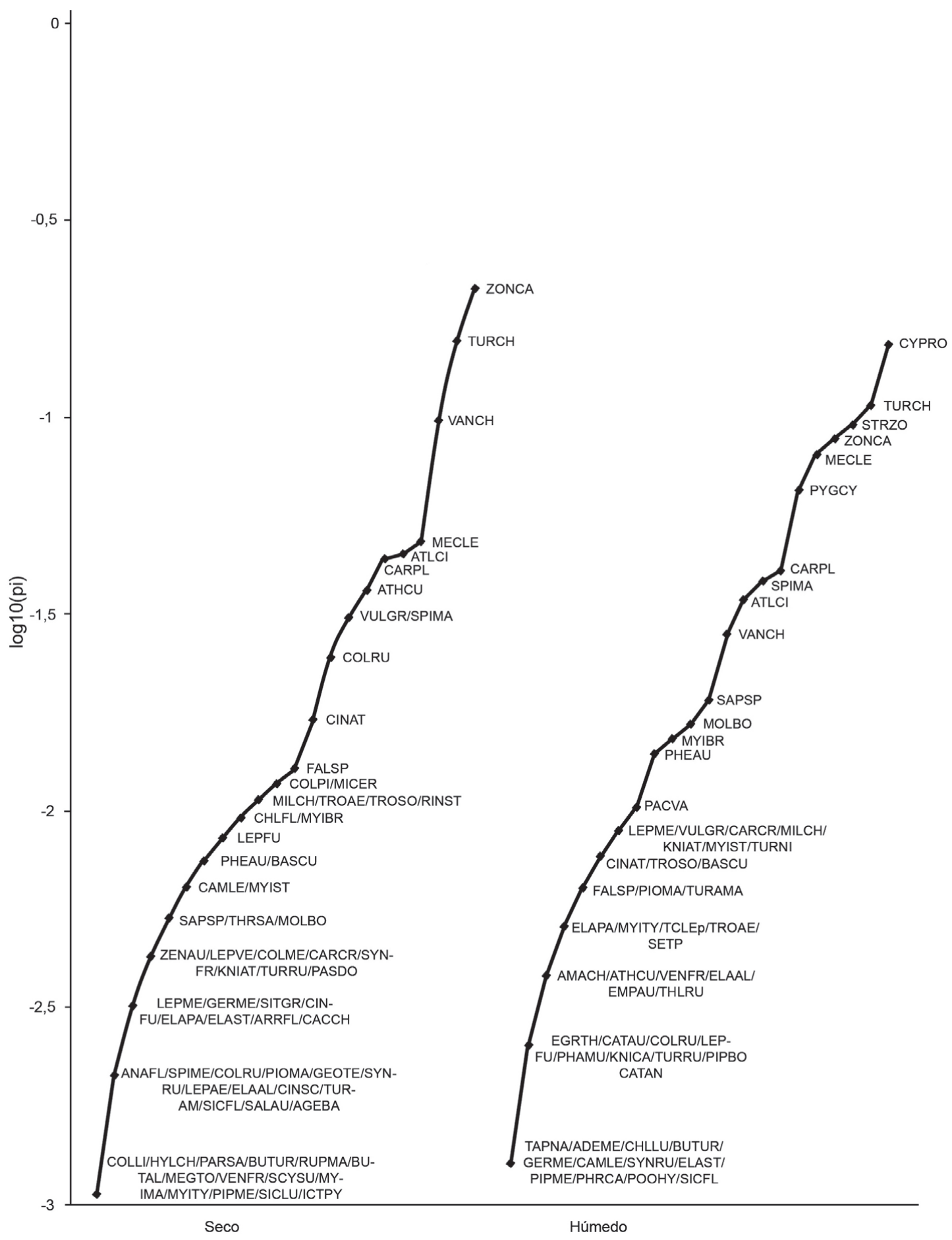

Figura 2. Curvas de rango-abundancia de las aves presentes en las Estaciones Seca y Húmeda, del Bosque Montano en la Quebrada del Portugués, Tucumán, Argentina. El código de las especies se detalla en la Tabla 1.

\section{DISCUSIÓN}

Los datos obtenidos revelan que el sector de Bosques Montanos de la Quebrada del Portugués resguarda una comunidad de aves de alto valor. Las 93 especies observadas 
corresponden al 19,3\% de la avifauna citada por Brandán y Navarro (2009) para la provincia. A su vez, 80 especies de las registradas en este estudio son típicas de las Yungas del NOA, lo que representa un 27,2\% del total de las especies citadas para esta provincia fitogoegráfica por Blendinger y Álvarez (2009).

La heterogeneidad estructural de los bosques montanos, debido a las distintas etapas sucesionales de sus parches, tanto de origen natural como antrópico (Ahumada et al., 2000; Brown et al., 2006), permitiría generar diferentes microambientes favorables para las aves. Esto explicaría el alto número de especies observadas, a pesar de que el área censada es un pequeño sector representativo de este tipo de ambiente.

El presente trabajo aportaría 47 especies nuevas al inventario disponible sobre las aves de Bosques Montanos de Tucumán (Vides Almonacid, 1985, 1992). Algunas de estas especies son de ambientes de humedales como: Anas flavirostris, Pardirallus sanguinolentus, Egretta thula y Megaceryle torquata, otras de bosques como: Colaptes melanochloros, Pionus maximiliani, Pipraeidea bonariensis, Synallaxis frontalis y otras de pastizales y matorrales: Colaptes rupicola y Leptasthenura aegithaloides.

En relación a los pisos altitudinales de las Yungas, según Vides Almonacid (1992), el Bosque Montano se caracteriza por ser el de menor riqueza de especies. Solamente reportó 73 especies para dichos bosques en la provincia de Tucumán y 49 especies para el área protegida Parque Sierra de San Javier. Esto sugeriría que la Quebrada del Portugués contiene una importante comunidad de aves de Bosque Montano.

Se destaca la presencia de Atlapetes citrinellus, Cinclus schulzii, Scytalopus superciliaris y Microspingus erythrophrys, quienes fueron indicadas por Stattersfield et al., (1998) como especies consideradas prioritarias de conservación para la región. Dicha clasificación se basa en el rango restringido de distribución y por sus números poblacionales reducidos o en disminución. Además, se observó la presencia de endemismos de Argentina como Scytalopus superciliaris, Phrygilus carbonarius y Atlapetes citrinellus (Capllonch, Ortiz, Nuñez Montellano, Blendinger, 2014), lo cual refuerza la importancia de esta zona como un ambiente de alta prioridad de conservación para la avifauna.

Moschione (2005), en su descripción del AICA Quebrada del Portugués, cita la presencia de ocho especies de importancia por ser consideradas Amenazadas a Nivel Mundial y/o Especies de Distribución Restringida, tales como: Vultur gryphus, Cypseloides rothschildi, Scytalopus superciliaris, Elaenia strepera, Cinclus schulzii, Atlapetes citrinellus, Amazona tucumana y Eriocnemis glaucopoides. Estas dos últimas, fueron las únicas especies no registradas en el presente estudio, por lo que es imperioso realizar nuevos muestreos para evaluar su presencia. Sin embargo, consideramos que tomar medidas de manejo inmediatas redundaría en la protección de las especies de alto valor de conservación mencionadas, así como también de todas aquellas especies típicas de las Yungas presentes en el área (Blendinger y Álvarez, 2009).

Se destaca la presencia de Adelomyia melanogenys, una especie de Trochilidae que frecuenta estratos bajos en los bosques y selvas de Yungas (López-Lanús, 2017). Dicho picaflor, de hábitos solitarios y ocultos, se caracteriza por tener una abundancia escasa y una distribución restringida sólo para las provincias de Salta y Jujuy. En Tucumán, existe sólo un registro documentado, en la zona de Raco, departamento 
Tafí Viejo (Ecoregistro 607641, 30 de agosto de 2017, Sebastián Rozadilla), por lo que el avistamiento en la Quebrada del Portugués representa el segundo registro para la provincia.

Las diferencias estacionales observadas en la abundancia relativa podrían deberse al aumento en el número de los individuos de alguna de las especies presentes en el período seco. En este período, las dominantes aportan más individuos que las del período húmedo, coincidiendo con que muchas especies presentaron una baja abundancia, lo que podría deberse a que muchas de ellas se desplazan en primaveraverano a otros pisos altitudinales para reproducirse (Capllonch, 1997). En la curva de rango abundancia, en el período húmedo se destaca un mayor arribo de especies migrantes latitudinales tales como: Empidonomus aurantioatrocristatus, Tachycineta leucopyga, Catamenia analis, Tapera naevia y Cypseloides rothschildi. En este sentido, los bosques montanos, podrían representar un lugar importante como sitios de paso o descanso para especies migratorias con desplazamientos largos (migrantes regionales), principalmente durante el período húmedo. Sin embargo, las especies residentes más abundantes (Zonotricia capensis, Turdus chiguanco y Vanellus chilensis) no mostraron diferencias estacionales, lo que podría indicar que la variación estacional no influiría en ellas, como lo hace en el resto de la comunidad, afectando tanto la abundancia como la permanencia en el Bosque Montano.

Es importante mencionar que, durante este estudio, se observó un fuerte impacto antrópico en la Quebrada, por ejemplo, el ingreso de vehículos, presencia de ganado de trashumancia, de residuos de pobladores cercanos y turistas y tala no selectiva. Esta falta de uso y seguimiento de pautas de manejo de los recursos podría afectar negativamente a la comunidad de aves presente. Si tenemos en cuenta que las aves son consideradas bioindicadoras de la calidad del ambiente (Noss 1990), es posible que otras comunidades de fauna y flora de la zona también se encuentren afectadas.

Si bien este es el primer estudio de base de la avifauna para la Quebrada del Portugués, consideramos que a partir de este trabajo se deberían establecer futuros estudios sistemáticos, y a una mayor escala temporal y espacial. Teniendo en cuenta el potencial biológico que tiene esta área para toda la región y sobre todo el hecho de que la misma forma parte del nuevo Parque Nacional Aconquija, es necesario contar con estudios de base sólidos para su futuro manejo y conservación.

\section{AGRADECIMIENTOS}

Agradecemos al Lic. Pablo Pereyra de la Sección Iconografía de la FML, por la elaboración del mapa. A los revisores y editores por sus comentarios y consejos, los cuales mejoraron el presente trabajo. 


\section{FINANCIAMIENTO}

El presente trabajo fue financiado por la Fundación Miguel Lillo y por la comuna del Mollar, Tucumán.

\section{PARTICIPACIÓN}

Todos los autores participaron en la toma de datos y análisis de los mismos, así como también la redacción del presente artículo.

\section{CONFLICTOS DE INTERÉS}

No existen conflictos de interés entre los autores o con terceros.

\section{LITERATURA CITADA / BIBLIOGRAPHY}

Ahumada, A. L., Ledesma, F. C., Ibañez Palacio, J. P., Delgado, M, Jiménez, M. J. (2000). Una evaluación de riesgo potencial en la región noreste de la Sierra del Aconquija, Tucumán, Argentina. IX Congreso Geológico Chileno, Actas $1,16-20$.

Antelo, C. M., Brandán Fernández, Z. J. (2013). Las aves no passeriformes de Tucumán. Argentina. Ministerio de Cultura y Educación, Fundación Miguel Lillo, Tucumán, Argentina. Miscelánea, 132.

Arturi, M. F., Grau, H. R., Aceñolaza, P. G., Brown, A. D. (1998). Estructura y sucesión en bosques montanos del Noroeste de Argentina. Revista de Biología Tropical, 46: 525-532.

Barros, J., Giménez, A. M. (2017). El valor de un bosque montano en Catamarca, Argentina. En Los bosques actuales del chaco semiárido argentino. Ecoanatomía y biodiversidad. Una mirada propositiva. Parte 1. Diversidad (105-126). Facultad de Ciencias Forestales, Univ. Nacional de Santiago del Estero, Argentina. Página. 279.

Bianchi, A. R., Cravero, S. A. C. (2010). Atlas climático digital de la República Argentina. Descripción climática mapas de temperatura media mensual y anual, precipitación anual, evapotranspiración potencial mensual y anual, balance hídrico directo anual, índice de áridez de Martone. INTA. https://inta.gob. ar/sites/default/files/script-tmp-texto_atlas_climtico_digital_de_la_argentina_ 110610 2.pdf.

Bibby, C. J., Hill, D. A., Burgess N. D. (2000). Bird Census Techniques. 2nd edn. Academic Press, London.

Blendinger, P. G., Álvarez, M. E. (2009). Aves de la Selva Pedemontana de las Yungas australes. En Selva Pedemontana de las Yungas. Historia natural, ecología 
y manejo de un ecosistema en peligro (233-272). Ediciones del Subtrópico, Tucumán, Argentina.

Brandán, Z. J., Navarro, C. I. (2009). Lista actualizada de las aves de la Provincia de Tucumán. Bird Checklist. Publicación especial, Fundación Miguel Lillo, Tucumán, Argentina.

Brown, A. D., Grau, H. R., Malizia, L. R., Grau, A. (2001). Argentina. En Bosques nublados del neotrópico (625-659). Instituto Nacional de Biodiversidad (INBio), Santo Domingo de Heredia, Costa Rica.

Brown, A. D., Pacheco, S., Lomáscolo, T., Malizia, L. R. (2006). Situación ambiental de los Bosques Andinos Yungueños. En La situación ambiental argentina 2005, (587), Fundación Vida Silvestre Argentina, Buenos Aires, Argentina.

Cabrera, A. L. (1976). Regiones Fitogeográficas Argentinas. Enciclopedia Argentina de Agricultura y Jardinería 2: 1-85. ACME. Buenos Aires, Argentina.

Canevari, M., Canevari, P., Carrizo, R., Harris, G., Rodríguez Mata, J., Straneck, R. J. (1991). Nueva guía de las aves argentinas. Tomos I y II. Fundación Acindar, Buenos Aires, Argentina.

Capllonch, P. (1997). La avifauna de los bosques de transición del noroeste argentino. Tesis Doctoral, Univ. Nacional de Tucumán, Tucumán, Argentina.

Capllonch, P., Lobo, R. (2005). Contribución al conocimiento de la migración de tres especies de Elaenia de Argentina. Ornitología Neotropical, 16, 145-161.

Capllonch, P., Ortiz, D., Soria, K. (2008). Migración del Zorzal común Turdus amaurochalinus (Turdidae) en Argentina. Revista Brasileira de Ornitología, 16, 12 22.

Capllonch, P., Ortiz, D., Montellano Nuñez. M. G., Blendinger. P. G. (2014). Aportes sobre la distribución, comportamiento y biología del cerquero amarillo, Atlapetes citrinellus (Aves: Emberizidae). Acta Zoológica Lilloana, 58, 222-240.

Chalukian, C. (1991). Regeneración, sucesión y plantas invasoras en un bosque Yungas, Salta, Argentina. Tesis de Magister. Universidad Nacional de Heredia, Costa Rica.

Chiesa, R. (2018). Aconquija, el parque nacional del Bicentenario. Aves Argentinas, $52,14-21$.

Cocimano, M. C., Chani, J. M., Echevarria. A. L., Marano, C. F. (2005). La comunidad de aves del Río Los Sosa, Tucumán-Argentina. XI Reunión Argentina de Ornitología. Buenos Aires.

Convención sobre el Comercio Internacional de Especies Amenazadas de Fauna y Flora Silvestres CITES. Apéndices I, II y III en vigor a partir del 4 de octubre de 2017. https://www.cites.org/esp/app/appendices.php

Echevarria, A. L. (2014). Las aves como indicadoras de problemas ambientales en el Embalse La Angostura, Tucumán, Argentina. Acta Zoológica Lilloana, 58, 44-56.

Echevarria, A. L., Marano, C. F., Chani, J. M., Cocimano, C. M. (2008). Composición de la comunidad de aves del Embalse La Angostura, Tafí del Valle, Tucumán, Argentina. Acta Zoológica Lilloana, 52, 98-105. 
Echevarria, A. L., Fanjul. M. E. (2016). Estructura, composición y variación estacional de la avifauna del embalse Escaba (selva montana subtropical), Tucumán, Argentina. Acta Zoológica Lilloana, 60, 101-115.

Fava, G. A., Acosta, J. C., Blanco, G. M. (2017). Efecto de la estacionalidad y lluvias en la avifauna del Chaco Serrano Austral, Argentina. Revista de Biología Tropical, 65, 953-961.

Feinsinger, P. (2003). El Diseño de estudios de Campo para la Conservación de la Biodiversidad. Editorial FAN. Santa Cruz de la Sierra, Bolivia. 155-157.

Grau, H. R. (2005). Dinámica de bosques en el gradiente altitudinal de las Yungas Argentinas. En Ecología y manejo de los bosques de Argentina. (1-30). La Plata, EDULP (Editorial de la Universidad de la Plata).

López Lanús, B. (2017). Guía Audiornis de las Aves de Argentina: Fotos y Sonidos, Identificación por características contrapuestas y marcas sobre imágenes. Segunda edición ampliada. Buenos Aires, Argentina: Copyright Audiornis Producciones.

López Lanús, B., Grilli, P., Coconier, E., Di Giacomo, A., Banchs, R. (2008). Categorización de las aves de la Argentina según su estado de conservación. Informe de Aves Argentinas (AOP) y Secretaría de Ambiente y Desarrollo Sustentable (SAyDS). Buenos Aires, Argentina.

Magurran, A. E., McGill. B. J. (2011). Biological diversity: Frontiers in measurement and assessment. Oxford University Press, Oxford.

Malizia, L. R., Aragón, R., Chacoff, N., Monmany, A. (1998). ¿Son las rutas una barrera para el desplazamiento de las aves? El caso de la Reserva Provincial La Florida (Tucumán, Argentina). El Hornero, 15, 10-16.

Malizia, L., Pacheco, S., Blundo, C., Brown, A. D. (2012). Caracterización altitudinal, uso y conservación de las Yungas Subtropicales de Argentina. Ecosistemas, 21, 53-73.

Mazar Barnett, J., Pearman. M. (2001). Lista comentada de las aves argentinas. Annotated checklist of the birds of Argentina. Lynx Edicions, Barcelona.

Moschione, F. (2005). Áreas importantes para la conservación de las aves en la Argentina. Sitios prioritarios para la conservación de la biodiversidad. Monografía de Aves Argentinas, 5, 488-489. Aves Argentinas/ Asociación Ornitológica del Plata, Buenos Aires.

Navarro, C. I., Brandán, Z. J., Marigliano, N. L. (2007). Lista comentada de las aves de un fragmento de la Reserva Provincial La Florida, Tucumán, Argentina. Acta Zoológica Lilloana, 51, 142-150.

Noss, R. F. (1990). Indicators for monitoring biodiversity: A hierarchical Approach. Conservation Biology, 4, 355-364.

Olrog, C. C. (1979). Nueva lista de la avifauna argentina. Opera Lilloana, 27, Fundación Miguel Lillo, Tucumán, Argentina.

Ortiz, D., Capllonch, P., Aveldaño, S., Mamaní, J., Quiroga, O., Moreno Ten, T. (2013). Los Passeriformes de Tucumán, Argentina: lista, distribución y migración. Rev. Boletín Biológica, 16, 39-71. Museo Prov. Cs. Nat. Florentino Ameghino. Buenos Aires, Argentina. 
Pérez Miranda, C. (2003).Tucumán y los Recursos Naturales. Biodiversidad Los Recursos Silvestres. Los Ambientes Naturales y las Áreas Protegidas. Gobierno de la Provincia de Tucumán, PROSAP (Programa de Servicios Agrícolas Provinciales), EPDA Tucumán (Entidad de Programación de Desarrollo Agropecuario) y Secretaria de Agricultura, Ganadería y Pesca de la Nación. Buenos Aires, Argentina.

Rappole, J. H. (1995). The Ecology of Migrant Birds. A Neotropical Perspective. Smithsonian Institution Press.

Remsen, J. V. Jr, Areta, J. I., Cadena, C. D., Claramunt, S., Jaramillo, A., Pacheco, J. F., Pérez-Emán, J., Robbins, M. B., Stiles, F. G. , Stotz, D. F., Zimmer, K. J. (2019). A classification of the bird species of South America. American Ornithologists'Union. http://www.museum.lsu.edu/ Remsen/SACCBaseline. htm.

Santillán, V., Quitia, M., Tinoco, B. A., Zárate, E., Schleuning, M., Böhning-Gaese, K., Neuschulz, E. L. (2018). Spatio-temporal variation in bird assemblages is associated with fluctuations in temperature and precipitation along a tropical elevational gradient. Plos One. https://doi.org/10.1371/journal.pone.01961 79.

Stattersfield, A. J., Crosby, M. J., Long, A. J., Wege, D. C. (1998). Endemic bird areas of the world. Priorities for conservation. Birdlife conservation series $n^{0}$ 7. BirdLife International, Cambridge, Reino Unido.

Tejedor Garavito, N., Álvarez, E., Arango Caro, S., Araujo Murakami, A., Blundo, C., Boza Espinoza, T. E., La Torre Cuadros, M. A., Gaviria, J., Gutiérrez, N., Jorgensen, P. M., León, B., López Camacho, R., Malizia, L., Millán, B., Moraes, M., Pacheco, S., Rey Benayas, J. M., Reynel, C., Timaná de la Flor, M., Ulloa, S., Vacas Cruz, O., Newton, A. C. (2012). Evaluación del estado de conservación de los bosques montanos en los Andes tropicales. Ecosistemas, 21, 148-166.

UICN. Unión Internacional para la conservación de la naturaleza. (Versión 2017-3). Red List of Threatened Species. http://www.iucnredlist.org/. Consultada 07 May 2018.

Vides Almonacid, R. (1985). Estudio comparativo de las taxocenosis de aves de los bosques de aliso de Tucumán. Tesis de licenciatura, Facultad de Ciencias Naturales, Univ. Nacional de Tucumán, Tucumán, Argentina.

Vides Almonacid, R. (1992). Estudio comparativo de las taxocenosis de aves de los bosques montanos de la Sierra de San Javier, Tucumán: bases para su manejo y conservación. Tesis doctoral, Univ. Nacional de Tucumán, Tucumán, Argentina.

Wiens, J. A. (1992). The Ecology of Bird Communities. Volume I. Foundations and Patterns. Cambridge University Press, Cambridge. 